\title{
Overview of the 2012 Revised International Chapel Hill Consensus Conference Nomenclature of Vasculitides
}

\author{
J. Charles Jennette, MD \\ Brinkhous Distinguished Professor and Chair, Room 308 Brinkhous-Bullitt Building, Department \\ of Pathology and Laboratory Medicine, University of North Carolina at Chapel Hill, Chapel Hill, \\ NC 27599, Phone: 919-966-4676 \\ J. Charles Jennette: jcj@med.unc.edu
}

\begin{abstract}
The nomenclature and classification of vasculitis has been difficult and controversial for many decades. This is problematic for both research on vasculitis as well as clinical care of patients with vasculitis. The first (1994) International Chapel Hill Consensus Conference on the Nomenclature of Systemic Vasculitides (CHCC) proposed names and definitions for the most common forms of vasculitis. Since then, there have been substantial advances in our understanding of vasculitis and changes in medical terminology. In addition, CHCC 1994 did not propose a nomenclature for some relatively common forms of vasculitis, such as vasculitis secondary to other diseases. To address these issues, a second International Chapel Hill Consensus Conference was held in 2012. The goals were to change names and definitions as appropriate, and add important categories of vasculitis not included in CHCC 1994. This overview summarizes the 2012 CHCC and points out the changes compared to the 1994 CHCC. Notable changes include the introduction of new terms such as granulomatosis with polyangiitis, eosinophilic granulomatosis with polyangiitis and $\operatorname{IgA}$ vasculitis, and the inclusion of categories for variable vessel vasculitis and secondary forms of vasculitis.
\end{abstract}

\section{Keywords}

Vasculitis; classification; Chapel Hill Consensus Conference

\section{Introduction}

The 1994 International Chapel Hill Consensus Conference on the Nomenclature of Systemic Vasculitides (CHCC 1994) proposed names and definitions for the most common forms of vasculitis [1]. This nomenclature was widely adopted. However, in the intervening years, there have been substantial advances in our understanding of vasculitis and a trend to reduce the use of eponyms in medical terminology. In addition, CHCC 1994 focused on a limited number of vasculitides, and thus did not propose a nomenclature for some relatively common forms of vasculitis, such as vasculitis secondary to other diseases. To address these issues, a second International Chapel Hill Consensus Conference was held in 2012 (CHCC

Disclosure: The author has declared no competing interest. 
2012) [2]. The goals were to change names and definitions as appropriate, and add important categories of vasculitis not included in CHCC 1994. This overview will summarize the proposals of the CHCC 2012 [2] (Table 1, Fig 1).

The product of the CHCC is a nomenclature system (nosology) and does not specify criteria by which patients are classified for clinical studies or diagnosed for clinical care. A nomenclature system provides names and definitions. Classification and diagnostic criteria are findings that are used to conclude that a specific category of disease is present in a specific patient. The definition of a disease provides the framework for developing classification and diagnostic criteria, but the criteria must be developed and validated by observations of cohorts of patients.

\section{Large vessel vasculitis (LVV)}

CHCC 2012 [2] defines LVV as vasculitis affecting the aorta and its major branches more often than other vasculitides, however, any size artery may be affected. This definition does not state that LVV predominantly affects large vessels because in many patients the number of medium and small arteries affected is greater than the number of large arteries affected. For example, only a few branches of the carotid arteries may be affected when there is involvement of numerous small branches extending into the head and neck, for example small ocular and periocular arteries. The two major variants of LVV are Takayasu arteritis (TA) and giant cell arteritis (GCA)

TA is arteritis, often granulomatous, predominantly affecting the aorta and its major branches [2]. Onset usually is in patients younger than 50. GCA is arteritis, often granulomatous, usually affecting the aorta and its major branches, with a predilection for the branches of the carotid and vertebral arteries. GCA often involves the temporal artery, and onset is usually in patients older than 50 and often associated with polymyalgia rheumatica. Thus, the major discriminator between TA and GCA is the age of the patient. This was concluded after substantial debate and careful review of available research and clinical data to try to identify other distinguishing features.

\section{Medium vessel vasculitis (MVV)}

MVV is vasculitis predominantly affecting medium arteries defined as the main visceral arteries and their branches. However, any size artery may be affected [2]. Two major categories are polyarteritis nodosa (PAN) and Kawasaki disease (KD).

PAN is necrotizing arteritis of medium or small arteries without glomerulonephritis or vasculitis in arterioles, capillaries, or venules; and not associated with ANCA [2]. The absence of ANCA is a valuable clinical feature in distinguishing PAN from microscopic polyangiitis [3]. KD is arteritis associated with the mucocutaneous lymph node syndrome and predominantly affecting medium and small arteries. Coronary arteries are often involved. KD usually occurs in infants and young children. 


\section{Small vessel vasculitis (SVV)}

SVV is divided into antineutrophil cytoplasmic antibody (ANCA)-associated vasculitis (AAV) and immune complex SVV [2]. This categorization was not in CHCC 1994 and reflects a greater level of confidence that ANCA are of pathogenic significance in AAV [4].

AAV is necrotizing vasculitis, with few or no immune deposits, predominantly affecting small vessels, associated with MPO-ANCA or PR3-ANCA [2]. A prefix should be added to indicate ANCA reactivity, e.g. PR3-ANCA, MPO-ANCA, ANCA-negative. This is important because mounting evidence indicates that the ANCA specificity identifies distinct categories of disease [5,6]. AAV is subdivided into microscopic polyangiitis (MPA), granulomatosis with polyangiitis (Wegener's) (GPA), and eosinophilic granulomatosis with polyangiitis (Churg-Strauss) (EGPA) [2]. The eponyms Wegener's granulomatosis and Churg-Strauss syndrome were replaced with the more descriptive terms GPA and EGPA respectively. The change to GPA was influenced by a decision that had already been made by several relevant medical societies [7].

MPA is necrotizing vasculitis, with few or no immune deposits, predominantly affecting small vessels [2]. Necrotizing arteritis involving small and medium arteries may be present. Necrotizing glomerulonephritis is very common. GPA is necrotizing granulomatous inflammation usually involving the upper and lower respiratory tract, and necrotizing vasculitis affecting predominantly small to medium vessels [2]. EGPA is eosinophil-rich and necrotizing granulomatous inflammation often involving the respiratory tract, and necrotizing vasculitis predominantly affecting small to medium vessels, and associated with asthma and eosinophilia [2]. ANCA is more frequent in EGPA when glomerulonephritis is present [8].

Immune complex SVV is vasculitis with moderate to marked vessel wall deposits of immunoglobulin and complement components predominantly affecting small vessels [2]. Glomerulonephritis is frequent. The categories of immune complex SVV that were included in CHCC 2012 are anti-glomerular basement membrane (anti-GBM) disease, cryoglobulinemic vasculitis (CV), IgA vasculitis (Henoch-Schönlein) (IgAV), and hypocomplementemic urticarial vasculitis (HUV) (anti-C1q vasculitis). Anti-GBM disease is vasculitis affecting glomerular capillaries, pulmonary capillaries, or both, with basement membrane deposition of anti-basement membrane autoantibodies [2]. This was included as an immune complex vasculitis because the pathogenesis involves in situ formation of immune complexes between anti-GBM antibodies and GBM antigens with resultant activation of inflammatory mediators. $\mathrm{CV}$ is vasculitis with cryoglobulin immune deposits affecting small vessels and associated with cryoglobulins in serum [2]. IgAV is vasculitis, with IgA1-dominant immune deposits, affecting small vessels [2]. Glomerulonephritis indistinguishable from IgA nephropathy may occur. The eponym Henoch-Schönlein was dropped because of the compelling evidence that this category of vasculitis is caused by abnormal IgA1 [9]. Hypocomplementemic urticarial vasculitis (HUV) was the least common immune complex vasculitis that was included. HUV is vasculitis accompanied by urticaria and hypocomplementemia affecting small vessels and associated with anti-C1q antibodies 
[2]. Glomerulonephritis, arthritis, obstructive pulmonary disease, and ocular inflammation are common.

\section{Variable vessel vasculitis (VVV)}

VVV is vasculitis with no predominant type of vessel involved that can affect vessels of any size (small, medium, and large) and type (arteries, veins, and capillaries) [2]. Vasculitides that are included in this category are Behcet's disease (BD) and Cogan's syndrome (CS), neither of which frequently affects the kidneys.

\section{Single-organ vasculitis (SOV)}

SOV is vasculitis in a single organ that has no features that indicate that it is a limited expression of a systemic vasculitis [2]. The involved organ and vessel type should be included in the name (e.g. cutaneous small vessel vasculitis, testicular arteritis, central nervous system vasculitis). Some patients originally diagnosed with SOV will develop additional disease manifestations that warrant re-defining the case as one of the systemic vasculitides (e.g. cutaneous arteritis later declaring itself as systemic polyarteritis nodosa, etc.). It is important for nephrologists to note that renal-limited ANCA-associated glomerulonephritis is a limited expression of a systemic vasculitis and thus is not considered a SOV.

\section{Vasculitis associated with systemic disease or probable etiology}

Vasculitis that is associated with and may be secondary to a systemic disease. The diagnosis should have a prefix specifying the associated systemic disease (e.g. rheumatoid vasculitis, lupus vasculitis, etc.) [2]. Likewise, vasculitis associated with a probable etiology should have a prefix specifying the association (e.g. hydralazine-associated MPA, hepatitis B virusassociated vasculitis, hepatitis $C$ virus-associated cryoglobulinemic vasculitis, etc.). These categories emphasize that a primary cause should always be sought in a patient with vasculitis.

\section{References}

1. Jennette JC, Falk RJ, Andrassy K, Bacon PA, Churg J, Gross WL, Hagen EC, Hoffman GS, Hunder GG, Kallenberg CGM, McCluskey RT, Sinico RA, Rees AJ, van Es LA, Waldherr R, Wiik A. Nomenclature of systemic vasculitides: The proposal of an international consensus conference. Arthritis Rheum. 1994; 37:187-192. [PubMed: 8129773]

2. Jennette JC, Falk RJ, Bacon PA, Basu N, Cid MC, Ferrario F, Flores-Suarez LF, Gross WL, Guillevin L, Hagen EC, Hoffman GS, Jayne DR, Kallenberg CGM, Lamprecht P, Langford CA, Luqmani RA, Mahr AD, Matteson EL, Merkel PA, Ozen S, Pusey CD, Rasmussen N, Rees AJ, Scott DGI, Specks U, Stone JH, Takahashi K, Watts RA. 2012 Revised International Chapel Hill Consensus Conference Nomenclature of Vasculitides. Arthritis Rheum. 2013; 65:1-11. [PubMed: 23045170]

3. Guillevin L, Lhote F, Amouroux J, Gherardi R, Callard P, Casassus P. Antineutrophil cytoplasmic antibodies, abnormal angiograms and pathological findings in polyarteritis nodosa and ChurgStrauss syndrome: indications for the classification of vasculitides of the polyarteritis Nodosa Group. Br J Rheumatol. 1996; 35:958-64. [PubMed: 8883433]

4. Jennette JC, Falk RJ, Hu P, Xiao H. Pathogenesis of Anti-neutrophil Cytoplasmic Autoantibody Associated Small Vessel Vasculitis. Annu Rev Pathol Mech Dis. 2013; 8:139-60. 
5. Lyons PA, Rayner TF, Trivedi S, Holle JU, Watts RA, Jayne DR, Baslund B, Brenchley P, Bruchfeld A, Chaudhry AN, Cohen Tervaert JW, Deloukas P, Feighery C, Gross WL, Guillevin L, Gunnarsson I, Harper L, Hrušková Z, Little MA, Martorana D, Neumann T, Ohlsson S, Padmanabhan S, Pusey CD, Salama AD, Sanders JS, Savage CO, Segelmark M, Stegeman CA, Tesař V, Vaglio A, Wieczorek S, Wilde B, Zwerina J, Rees AJ, Clayton DG, Smith KG. Genetically distinct subsets within ANCA-associated vasculitis. N Engl J Med. 2012; 367:214-23. [PubMed: 22808956]

6. Lionaki S, Blyth ER, Hogan SL, Hu Y, Senior JBA, Jennette CE, Nachman PH, Jennette JC, Falk RJ. Classification of antineutrophil cytoplasmic autoantibody vasculitides: The role of antineutrophil cytoplasmic autoantibody specificity for myeloperoxidase or proteinase 3 in disease recognition and prognosis. Arthritis Rheum. 2012; 64:3452-62. [PubMed: 23023777]

7. Falk RJ, Gross WL, Guillevin L, Hoffman G, Jayne DRW, Jennette JC, et al. "Granulomatosis with polyangiitis (Wegener's)": an alternative name for "Wegener's granulomatosis". A joint proposal of the American College of Rheumatology, the American Society of Nephrology, and the European League Against Rheumatism. Ann Rheum Dis. 2011; 70:704. [PubMed: 21372195] J Am Soc Nephrol. 2011; 22:587-8. [PubMed: 21372208] Arth Rheum. 2011; 63:863-4. [PubMed: 21374588]

8. Sinico RA, Di Toma L, Maggiore U, Tosoni C, Bottero P, Sabadini E, et al. Renal involvement in Churg-Strauss syndrome. Am J Kidney Dis. 2006; 47:770-9. [PubMed: 16632015]

9. Suzuki H, Kiryluk K, Novak J, Moldoveanu Z, Herr AB, Renfrow MB, et al. The pathophysiology of IgA nephropathy. J Am Soc Nephrol. 2011; 22:1795-803. [PubMed: 21949093] 


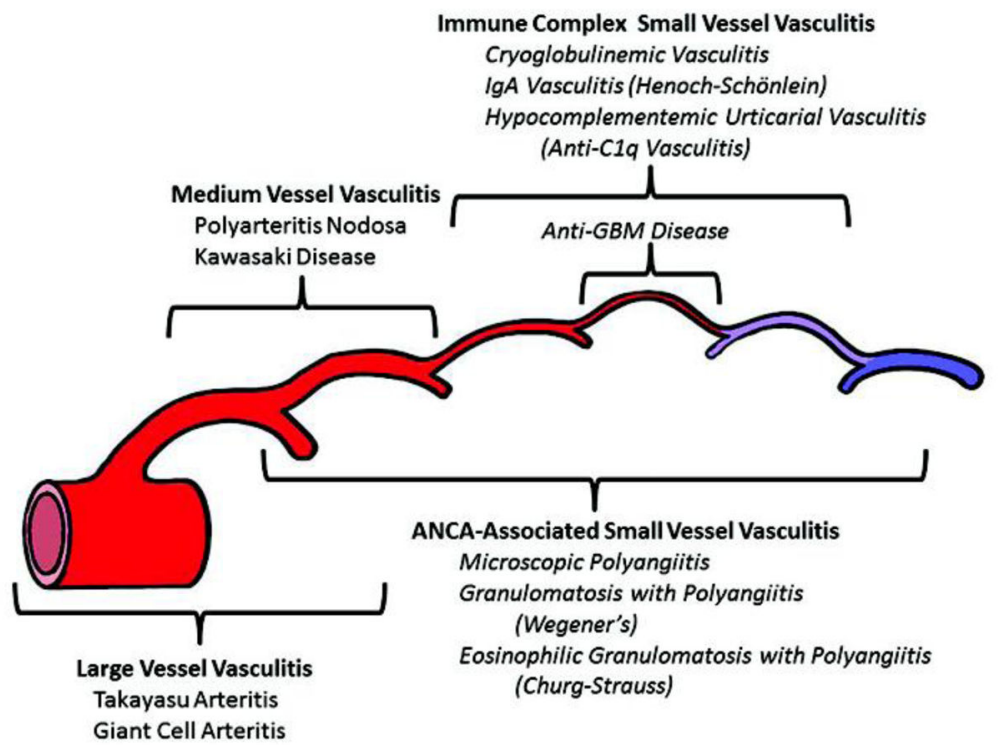

Fig 1.

Diagram depicting the usual distribution of vessel involvement by large vessel vasculitis, medium vessel vasculitis, and small vessel vasculitis. All 3 major categories of vasculitis can affect any size artery, although large vessel vasculitis most often affects large arteries. Medium vessel vasculitis predominantly affects medium arteries but small arteries may be affected. Small vessel vasculitis predominantly affects venules and capillaries. Immune complex small vessel vasculitis rarely affects arteries. Note that ANCA (antineutrophil cytoplasmic antibody) associated vasculitis affects a broader spectrum of vessels than immune complex vasculitis. Reproduced from reference 2 with permission. 


\section{Table 1}

Names for vasculitides adopted by the 2012 International Chapel Hill Consensus Conference on the Nomenclature of Vasculitides. The items highlighted in red are changes or additions compared to the 1994 International Chapel Hill Consensus Conference on the Nomenclature of Vasculitides. Modified from reference 2 .

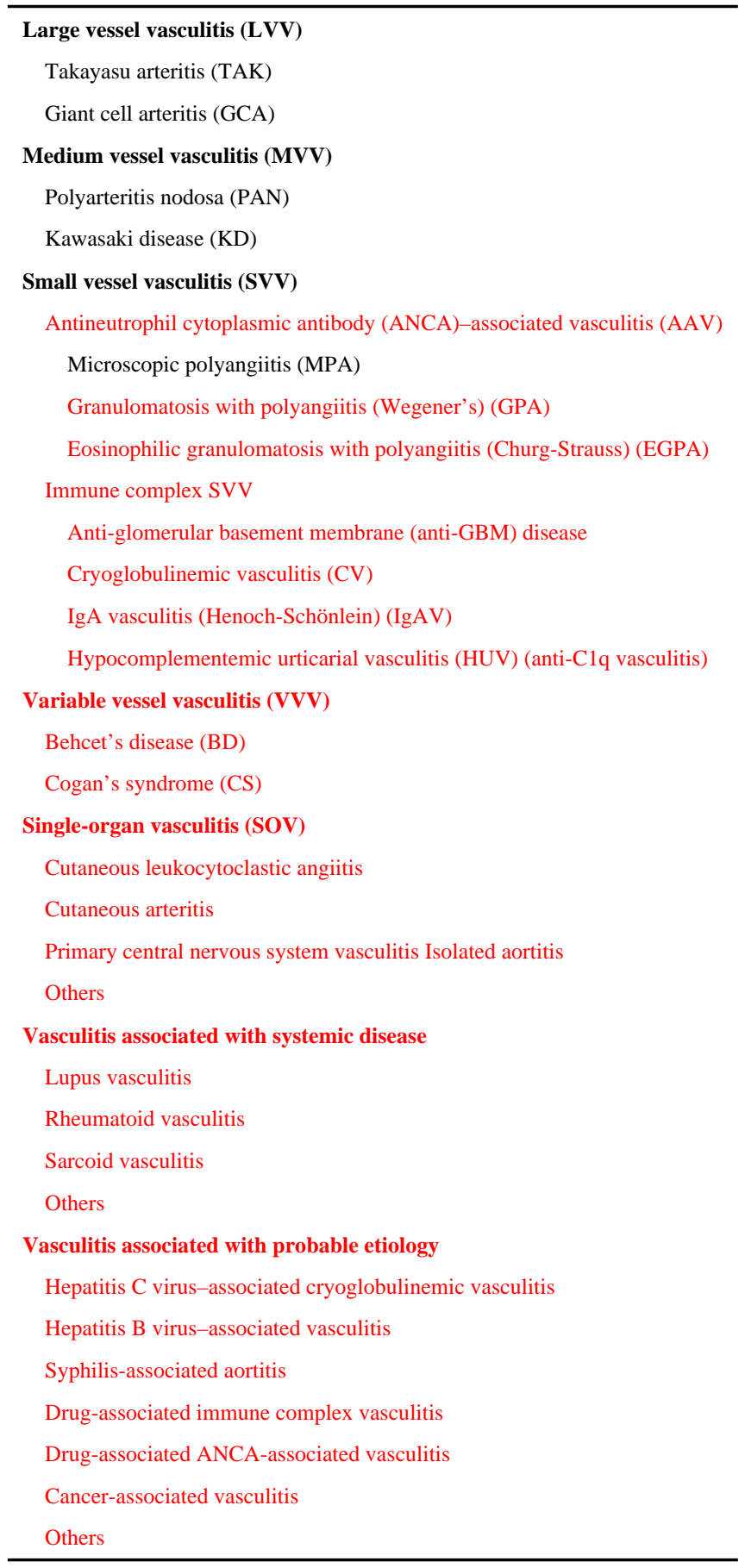

